\title{
Enantioselective ring expansion of prolinols and ring-closing metathesis: formal synthesis of $(-)$-swainsonine
}

\author{
Ingrid Déchamps, Domingo Gomez Pardo, and Janine Cossy* \\ Laboratoire de Chimie Organique associé au CNRS, \\ Ecole Supérieure de Physique et de Chimie Industrielles de la Ville de Paris (ESPCI), \\ 10 rue Vauquelin, 75231 Paris cedex 05, France \\ E-mail: janine.cossy@espci.fr
}

Dedicated to Pr. Tietze on the occasion of his $65^{\text {th }}$ birthday

\begin{abstract}
An efficient enantioselective formal synthesis of (-)-swainsonine has been achieved in 14 steps with 14\% global yield using an enantioselective ring enlargement of a substituted prolinol and a ring-closing metathesis as the key steps.
\end{abstract}

Keywords: Asymmetric synthesis, ring expansion, ring-closing metathesis, (-)-swainsonine

\section{Introduction}

The (1S,2R,8R,8aR)-1,2,8-trihydroxyindolizidine, (-)-swainsonine (Figure 1) belongs to the indolizidine class of alkaloid natural products. This compound has attracted the attention of synthetic chemists due to its interesting structure and potent biological properties. (-)-Swainsonine has been first isolated from the fungus Rhizoctonia leguminicola in 1973, ${ }^{1}$ and has been later isolated from other plants ${ }^{2}$ and fungus. ${ }^{3}$ (-)-Swainsonine has been the subject of many biological investigations and was found to be an effective inhibitor of both lysosomal $\alpha$-mannosidase ${ }^{4}$ and mannosidase II. $^{5}$ It has also demonstrated anticancer, ${ }^{6}$ anti-tumor proliferative $^{7}$ and immunoregulating activity. ${ }^{8}$ Since the first total syntheses in $1984,{ }^{9-12}$ there have been over 35 syntheses. ${ }^{13,14}$ In most of them, the starting material is a carbohydrate, which allows the introduction of the asymmetry and the control of the four asymmetric centers. By using D-erythrose, (-)-swainsonine could be synthesized in 8 steps, which is, to the best of our knowledge, the shortest synthesis up to now. ${ }^{15}$ A multigram scale synthesis in 10 steps from inexpensive D-ribose was also described recently. ${ }^{16}$ 


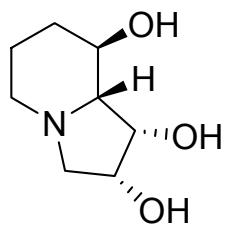

\section{Figure 1}

Except in the synthesis of swainsonine analogues, $\alpha$-amino acids or derivatives have not been used to synthesize (-)-swainsonine. Here, we would like to report a formal synthesis of $(-)$-swainsonine starting from L-proline. The two key steps are an enantioselective ring enlargement of a substituted prolinol into a 3-hydroxypiperidine ${ }^{17}$ and a ring-closing metathesis. ${ }^{18}$

The synthesis of (-)-swainsonine was envisaged from the unsaturated bicyclic amino compound $\mathbf{A}$, which would be synthesized by ring-closing metathesis applied to the amino diene B. This latter compound would be obtained by applying an enantioselective ring expansion to prolinol $\mathbf{C}$, which will be synthesized by a diastereoselective addition of an organometallic species on prolinal D. This latter compound would be obtained from L-proline (Scheme 1).

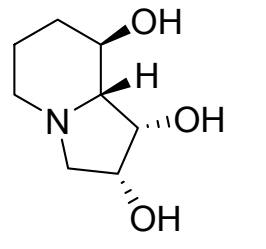

(-)-Swainsonine<smiles>O=C(O)C1CCCN1</smiles>

L-proline

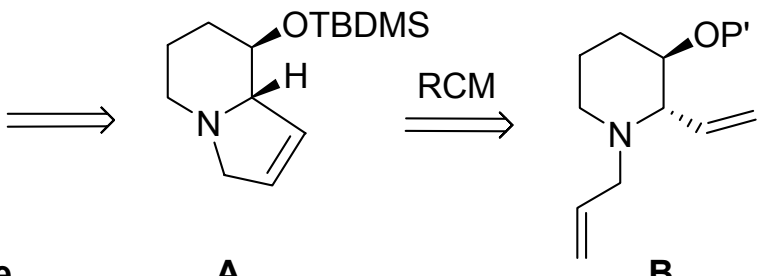

A

\section{B}

\section{Scheme 1}

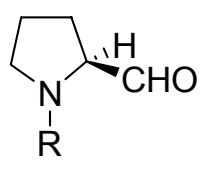

D
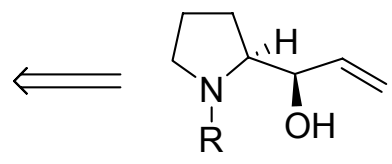

C

\section{Results and Discussion}

The synthesis of (-)-swainsonine started from L-proline, which was transformed in five steps into prolinol 4. ${ }^{19}$ After esterification $\left(\mathrm{SOCl}_{2}, \mathrm{MeOH}\right)$ and $\mathrm{N}$-alkylation by using trityl chloride, amino ester 1 was isolated in $90 \%$ yield. This latter compound was converted into prolinol 2 in 98\% yield by reduction using $\mathrm{LiAlH}_{4}$ in THF. After oxidation under Swern conditions (oxalyl 
chloride, DMSO, $\mathrm{Et}_{3} \mathrm{~N}, \mathrm{CH}_{2} \mathrm{Cl}_{2}$ ), aldehyde 3 was isolated in $95 \%$ yield and treated with vinylmagnesium chloride in ether to give the allylic alcohol 4 as a single isomer (93\% yield). We have to point out that the presence of the $N$-trityl group is of importance, as aldehydes of type D $\left(\mathrm{R}=\right.$ alkyl, benzyl, Boc, $\left.\mathrm{Cbz}, \mathrm{CO}_{2} \mathrm{Me}\right)$ are not configurationally stable in the presence of Grignard reagent, and the addition of organometallic onto these compounds usually led to a low diastereoselectivity. ${ }^{20}$ On the contrary, $N$-tritylprolinal $\mathbf{3}$ is stable and addition of various nucleophiles is achieved with high level of diastereoselectivity. ${ }^{19}$ In order to build up the 5 -membered ring of (-)-swainsonine by using a ring-closing metathesis, the $N$-trityl group in prolinol 4 was removed $\left(\mathrm{HCl}, \mathrm{Et}_{2} \mathrm{O}\right)$ and replaced by a $\mathrm{N}$-allyl group (Allyl-Br, $\mathrm{K}_{2} \mathrm{CO}_{3}$, toluene) to produce the substituted prolinol 5 (50\% yield), which is the precursor of the 3-hydroxypiperidine 6. In agreement with previous results on the ring expansion of substituted prolinols, ${ }^{21}$ the enantioselective ring enlargement of prolinol 5 to piperidine $\mathbf{6}$ was performed by using trifluoroacetic anhydride (TFAA), $\mathrm{Et}_{3} \mathrm{~N}$ in THF and then $\mathrm{NaOH}$. Under these conditions, 3-hydroxypiperidine 6 was isolated in 95\% yield with a diastereomeric excess superior to $95 \%$. After protection of the hydroxyl group in compound 6 (TBDMSCl, $\mathrm{Et}_{3} \mathrm{~N}, \mathrm{DMAP}, \mathrm{CH}_{2} \mathrm{Cl}_{2}$ ), piperidine 7 was isolated in $70 \%$ yield. As ruthenium catalysts are deactivated by amino groups, piperidine 7 was transformed to the ammonium salt $\mathbf{7}$, by treatment with camphorsulfonic acid (CSA) and then treated with commercially available Grubbs catalyst first generation ${ }^{22}$ (5 mol. \%) in refluxing $\mathrm{CH}_{2} \mathrm{Cl}_{2}$ and, after $6 \mathrm{~h}$, the reaction mixture was treated with $\mathrm{K}_{2} \mathrm{CO}_{3}$ to afford unsaturated bicyclic compound $\mathbf{8}$ in $82 \%$ yield (Scheme 2). The spectroscopic data of this latter compound are in agreement with those previously reported in the literature. ${ }^{23}$ This approach constitutes a formal synthesis of (-)-swainsonine in 14 steps as the transformation of compound 8 into (-)-swainsonine was described in 4 steps in the literature. ${ }^{23 \mathrm{~b}}$

In conclusion, we have developed a short synthesis of (-)-swainsonine in $14 \%$ overall yield from L-proline by using a highly diastereoselective addition of vinyl Grignard reagent on $N$-tritylprolinal, an enantioselective ring expansion of a substituted prolinol into a 3-hydroxypiperidine to build up the 6-membered ring and a final ring-closing metathesis to construct the 5-membered ring. Further applications of this approach to swainsonine analogues and biological testing are ongoing. 


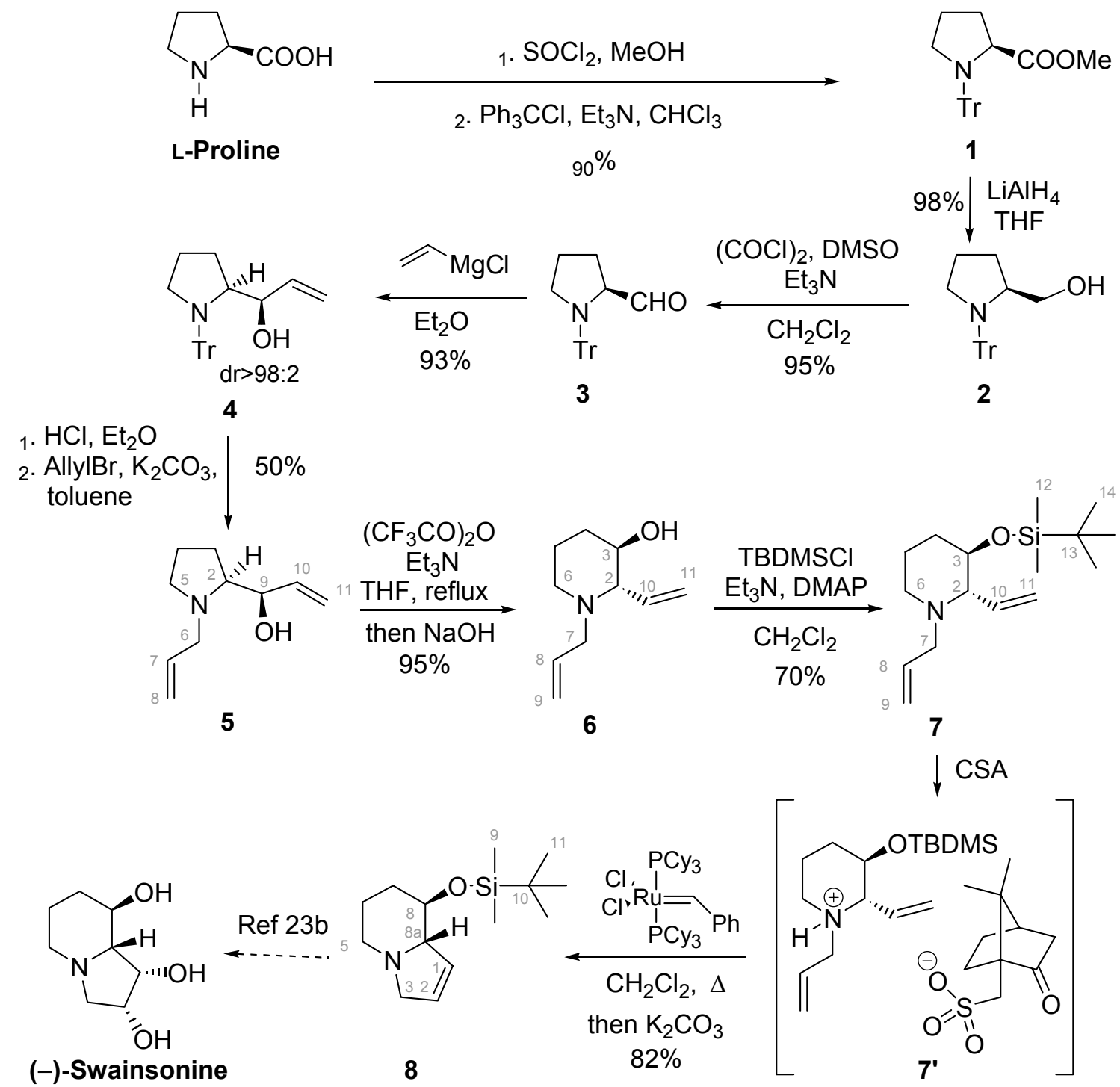

Scheme 2

\section{Experimental Section}

General Procedures. Commercially available reagents and solvents were used as received. Anhydrous solvents were distilled. Tetrahydrofuran and diethyl ether were purified by distillation from sodium and benzophenone, methylene chloride was dried by distillation from $\mathrm{CaH}_{2}$. TLC was performed on Merck $60 \mathrm{~F}_{254}$ silica gel plates and visualized either with a UV lamp $(254 \mathrm{~nm})$, or by using a solution of $\mathrm{KMnO}_{4} / \mathrm{K}_{2} \mathrm{CO}_{3} / \mathrm{NaOH}$ in water followed by heating. Flash chromatography was performed with Merck Geduran Si60 silica gel (40-63 $\mu \mathrm{m})$. Infrared (IR) spectra were recorded on a Bruker TENSOR ${ }^{\mathrm{TM}} 27$ (IRFT); wavenumbers are indicated in $\mathrm{cm}^{-1}$. ${ }^{1} \mathrm{H}-\mathrm{NMR}$ spectra were recorded on a Bruker AVANCE 400 at $400 \mathrm{MHz}$ and data are 
reported as follows: chemical shift in ppm from tetramethylsilane as an internal standard, multiplicity $(\mathrm{s}=$ singlet, $\mathrm{d}=$ doublet, $\mathrm{t}=$ triplet, $\mathrm{q}=$ quartet, $\mathrm{m}=$ multiplet $)$, integration, assignment. ${ }^{13} \mathrm{C}-\mathrm{NMR}$ spectra were recorded on a Bruker AVANCE 400 at $100 \mathrm{MHz}$ and data are reported as follows: chemical shift in ppm from tetramethylsilane with the solvent as an internal indicator $\left(\mathrm{CDCl}_{3} \delta 77.0 \mathrm{ppm}\right)$, multiplicity with respect to proton (deduced from DEPT experiments, $\mathrm{s}=$ quaternary $\mathrm{C}, \mathrm{d}=\mathrm{CH}, \mathrm{t}=\mathrm{CH}_{2}, \mathrm{q}=\mathrm{CH}_{3}$ ), assignment. Mass spectra with electronic impact (MS-EI) were recorded from a Hewlett-Packard tandem 5890A GC (12 m capillary column) - 5971 MS (70 eV). Mass spectra with chemical ionization (MS-CI) and high resolution mass spectra (HRMS) were performed by the Centre de Spectrochimie Organique de l'Ecole Normale Supérieure Ulm (Paris). Optical rotations were measured on a Perkin-Elmer 343 polarimeter in a $10 \mathrm{~cm}$ cell.

(R)-1-[(S)-1-Allyl-pyrrolidin-2-yl]-prop-2-en-1-ol (5). To a stirred solution of prolinol $\mathbf{4}^{19}$ (5.33 g, $14.4 \mathrm{mmol}, 1.0$ equiv) in $\mathrm{Et}_{2} \mathrm{O}(40 \mathrm{~mL})$ at $0{ }^{\circ} \mathrm{C}$, was added $\mathrm{HCl}$ ( $5 \mathrm{M}$ aqueous solution, $29 \mathrm{~mL}$ ) dropwise. The cooling bath was removed and after $24 \mathrm{~h}$ of vigorous stirring at RT, the two phases were separated and the aqueous phase was washed three times by $\mathrm{Et}_{2} \mathrm{O}(3 \times 30 \mathrm{~mL})$. Toluene $(150 \mathrm{~mL})$ was added to the aqueous phase, the mixture was cooled down to $0{ }^{\circ} \mathrm{C}$, and $\mathrm{K}_{2} \mathrm{CO}_{3}(26 \mathrm{~g})$, then tetrabutylammonium bromide $(0.93 \mathrm{~g}, 2.9 \mathrm{mmol}, 0.2$ equiv) and allyl bromide (3.75 mL, $43.3 \mathrm{mmol}, 3.0$ equiv) were added to the reaction mixture. After $24 \mathrm{~h}$ of vigorous stirring at $\mathrm{RT}$, the two phases were separated. The aqueous phase was extracted twice with ethyl acetate $(2 \times 50 \mathrm{~mL})$. The organic phases were dried over $\mathrm{Na}_{2} \mathrm{SO}_{4}$ and the solvents were evaporated to dryness in vacuo. The crude product was purified by flash chromatography on silica gel (cyclohexane/Et $\left.{ }_{2} \mathrm{O}: 50 / 50\right)$. Compound 5 was obtained as a yellow oil (1.2 g, $7.2 \mathrm{mmol}$, 50\% yield). ${ }^{1} \mathrm{H}-\mathrm{NMR}\left(\mathrm{CDCl}_{3}, 400 \mathrm{MHz}\right): \delta 1.58-1.72\left(3 \mathrm{H}, \mathrm{H}_{3}\right.$ and $\left.\mathrm{H}_{4}\right), 1.82\left(\mathrm{~m}, 1 \mathrm{H}, \mathrm{H}_{3},\right), 2.30$ $\left(\mathrm{m}, 1 \mathrm{H}, \mathrm{H}_{5}\right), 2.53\left(\mathrm{~m}, 1 \mathrm{H}, \mathrm{H}_{2}\right), 2.92\left(\mathrm{dd}, J=13.7,7.5 \mathrm{~Hz}, 1 \mathrm{H}, \mathrm{H}_{6}\right), 3.15\left(\mathrm{~m}, 1 \mathrm{H}, \mathrm{H}_{5}\right.$ ), 3.36 (bs, $1 \mathrm{H}, \mathrm{OH}), 3.48\left(\mathrm{dd}, J=13.7,5.2 \mathrm{~Hz}, 1 \mathrm{H}, \mathrm{H}_{6}\right), 4.24\left(\mathrm{~m}, 1 \mathrm{H}, \mathrm{H}_{9}\right), 5.08-5.24\left(\mathrm{~m}, 3 \mathrm{H}, \mathrm{H}_{8}\right.$ and $\left.\mathrm{H}_{11}\right)$, 5.33 (ddd, $J=17.1,1.8,1.8 \mathrm{~Hz}, 1 \mathrm{H}, \mathrm{H}_{11}$ ), 5.77 (ddd, $J=16.9,10,7,5.4 \mathrm{~Hz}, 1 \mathrm{H}, \mathrm{H}_{10}$ ), 5.89 (dddd, $\left.J=17.2,10.0,7.5,5.5 \mathrm{~Hz}, 1 \mathrm{H}, \mathrm{H}_{7}\right) .{ }^{13} \mathrm{C}-\mathrm{NMR}\left(\mathrm{CDCl}_{3}, 100 \mathrm{MHz}\right): \delta 23.4\left(\mathrm{t}, \mathrm{C}_{4}\right), 24.0\left(\mathrm{t}, \mathrm{C}_{3}\right), 54.3$ $\left(\mathrm{t}, \mathrm{C}_{5}\right), 56.5\left(\mathrm{t}, \mathrm{C}_{6}\right), 67.2\left(\mathrm{~d}, \mathrm{C}_{2}\right), 69.8\left(\mathrm{~d}, \mathrm{C}_{9}\right), 115.4\left(\mathrm{t}, \mathrm{C}_{11}\right), 116.9\left(\mathrm{t}, \mathrm{C}_{8}\right), 135.8\left(\mathrm{~d}, \mathrm{C}_{7}\right), 137.7$ (d, $\mathrm{C}_{10}$ ). IR (neat): 3405, 3075, 2960, 2795, 1642, 1604, 1444, 1419, 1351, 1280, 1205, 1150, 992, $921 \mathrm{~cm}^{-1}$. MS (CI, $\left.\mathrm{CH}_{4}\right): m / z$ (relative intensity): $168\left(\mathrm{MH}^{+}, 100\right), 128$ (8), 150 (12), $126(4), 110$ (47), $98(3) .[\alpha]_{\mathrm{D}}{ }^{20}=-54.3\left(c 1.0, \mathrm{CHCl}_{3}\right)$. HRMS: Found: $m / z$ 168.1390. Calcd for $\mathrm{C}_{10} \mathrm{H}_{18} \mathrm{NO}$ : $(\mathrm{MH})^{+} 168.1388$.

(2S,3R)-1-Allyl-2-vinylpiperidin-3-ol (6). Trifluoroacetic anhydride (1.5 mL, $10.6 \mathrm{mmol}, 1.5$ equiv.) was added dropwise to a solution of prolinol 5 (1.2 g, $7.1 \mathrm{mmol}, 1.0$ equiv) in THF (60 $\mathrm{mL})$ at $0{ }^{\circ} \mathrm{C}$. After $1 \mathrm{~h}, \mathrm{Et}_{3} \mathrm{~N}(3.0 \mathrm{~mL}, 21.2 \mathrm{mmol}, 3.0$ equiv) was added dropwise, and the reaction mixture was stirred for $20 \mathrm{~min}$ at $0{ }^{\circ} \mathrm{C}$ and then heated to reflux for $15 \mathrm{~h}$. After addition of $\mathrm{NaOH}(2.5 \mathrm{M}$ aqueous solution, $15 \mathrm{~mL})$, the mixture was stirred for $2 \mathrm{~h}$ at $\mathrm{RT}$ and then extracted with EtOAc $(3 \times 30 \mathrm{~mL})$, dried over $\mathrm{Na}_{2} \mathrm{SO}_{4}$, and evaporated to dryness in vacuo. The residue was purified by flash column chromatography on silica gel (cyclohexane/EtOAc: 80/20) to give piperidine $6(1.1 \mathrm{~g}, 6.7 \mathrm{mmol}, 95 \%$ yield $)$ as a yellow oil. ${ }^{1} \mathrm{H}-\mathrm{NMR}\left(\mathrm{CDCl}_{3}, 400 \mathrm{MHz}\right): \delta$ 
$1.30\left(\mathrm{~m}, 1 \mathrm{H}, \mathrm{H}_{4}\right), 1.56\left(\mathrm{~m}, 1 \mathrm{H}, \mathrm{H}_{5}\right), 1.71\left(\mathrm{~m}, 1 \mathrm{H}, \mathrm{H}_{5}\right), 1.98-2.04\left(2 \mathrm{H}, \mathrm{H}_{4}\right.$, and $\left.\mathrm{H}_{6}\right), 2.23(\mathrm{bs}, 1 \mathrm{H}$, $\mathrm{OH}), 2.50\left(\mathrm{dd}, J=8.4,8.4 \mathrm{~Hz}, 1 \mathrm{H}, \mathrm{H}_{2}\right), 2.76-2.90\left(2 \mathrm{H}, \mathrm{H}_{6}\right.$, and $\left.\mathrm{H}_{7}\right), 3.31-3.39\left(2 \mathrm{H}_{,} \mathrm{H}_{3}\right.$ and $\left.\mathrm{H}_{7}\right)$, 5.10-5.19 (2H, $\left.\mathrm{H}_{9}\right), 5.31-5.37\left(2 \mathrm{H}, \mathrm{H}_{11}\right), 5.70-5.90\left(2 \mathrm{H}, \mathrm{H}_{8}\right.$ and $\left.\mathrm{H}_{10}\right) .{ }^{13} \mathrm{C}-\mathrm{NMR}\left(\mathrm{CDCl}_{3}, 100\right.$ MHz): $\delta 22.7\left(\mathrm{t}, \mathrm{C}_{5}\right), 30.8\left(\mathrm{t}, \mathrm{C}_{4}\right), 50.9\left(\mathrm{t}, \mathrm{C}_{6}\right), 58.3\left(\mathrm{t}, \mathrm{C}_{7}\right), 69.9\left(\mathrm{~d}, \mathrm{C}_{3}\right), 73.1\left(\mathrm{~d}, \mathrm{C}_{2}\right), 117.8\left(\mathrm{t}, \mathrm{C}_{9}\right)$, 120.3 (t, $\left.\mathrm{C}_{11}\right), 134.8$ (d, $\left.\mathrm{C}_{8}\right), 137.9$ (d, $\mathrm{C}_{10}$ ). IR (neat): 3415, 3076, 2936, 2861, 2793, 1643, 1441, 1419, 1261, 1090, 994, 916, $889 \mathrm{~cm}^{-1}$. MS (EI, 70eV) $\mathrm{m} / z$ (relative intensity): $167\left(\mathrm{M}^{+}, 9\right), 140$ (9), 126 (100), 122 (16), 110 (17), 108 (21), 96 (14), 94 (18), 82 (17), 71 (16), 68 (18), 56 (14). $[\alpha]_{\mathrm{D}}{ }^{20}=+50.2\left(c\right.$ 1.03, $\left.\mathrm{CHCl}_{3}\right)$. HRMS: Found: $m / z$ 168.1384. Calcd for $\mathrm{C}_{10} \mathrm{H}_{18} \mathrm{NO}:(\mathrm{MH})^{+}$ 168.1388 .

(2S,3R)-1-Allyl-3-(tert-butyldimethylsilanyloxy)-2-vinylpiperidine (7). To a solution of piperidine $6\left(0.670 \mathrm{~g}, 4.0 \mathrm{mmol}, 1.0\right.$ equiv) in $\mathrm{CH}_{2} \mathrm{Cl}_{2}(50 \mathrm{~mL})$ at RT, DMAP $(0.049 \mathrm{~g}, 0.4$ mmol, 0.1 equiv), $\mathrm{Et}_{3} \mathrm{~N}$ (1.13 mL, $8.0 \mathrm{mmol}, 2.0$ equiv) and TBDMSCl (1.20 g, $8.0 \mathrm{mmol}, 2.0$ equiv) were successively added. After $18 \mathrm{~h}$ at RT, the reaction mixture was quenched with a saturated aqueous $\mathrm{NaHCO}_{3}$ solution until $\mathrm{pH} \sim 10$. The aqueous layer was extracted with $\mathrm{CH}_{2} \mathrm{Cl}_{2}$ $(2 \times 30 \mathrm{~mL})$ and the combined organic phases were dried over $\mathrm{Na}_{2} \mathrm{SO}_{4}$ and filtered. The solvent was removed in vacuo. The crude oil was purified by flash column chromatography on silica gel (cyclohexane/EtOAc: $90 / 10)$ to give $7\left(0.788 \mathrm{~g}, 2.8 \mathrm{mmol}, 70 \%\right.$ yield) as a colorless oil. ${ }^{1} \mathrm{H}$ NMR $\left(\mathrm{CDCl}_{3}, 400 \mathrm{MHz}\right): \delta-0.02\left(\mathrm{~s}, 3 \mathrm{H}, \mathrm{H}_{12}\right), 0,00\left(\mathrm{~s}, 3 \mathrm{H}, \mathrm{H}_{12}\right), 0.83\left(\mathrm{~s}, 9 \mathrm{H}, \mathrm{H}_{14}\right), 1.28(\mathrm{~m}, 1 \mathrm{H}$, $\left.\mathrm{H}_{4}\right), 1.52\left(\mathrm{~m}, 1 \mathrm{H}, \mathrm{H}_{5}\right), 1.65\left(\mathrm{~m}, 1 \mathrm{H}, \mathrm{H}_{5}\right), 1.88-1.95\left(2 \mathrm{H}, \mathrm{H}_{4}\right.$, and $\left.\mathrm{H}_{6}\right), 2.42(\mathrm{dd}, J=8.6,8.6 \mathrm{~Hz}$, $\left.1 \mathrm{H}, \mathrm{H}_{2}\right), 2.74\left(\mathrm{dd}, J=13.9,8.0 \mathrm{~Hz}, 1 \mathrm{H}, \mathrm{H}_{7}\right), 2.88\left(\mathrm{~m}, 1 \mathrm{H}, \mathrm{H}_{6}\right), 3.35-3.49\left(2 \mathrm{H}_{,} \mathrm{H}_{3}\right.$ and $\left.\mathrm{H}_{7^{\prime}}\right)$, 5.07-5.14 (2H, $\left.\mathrm{H}_{9}\right), 5.19\left(\mathrm{dd}, J=4.4,1.9 \mathrm{~Hz}, 1 \mathrm{H}, \mathrm{H}_{11}\right), 5.22\left(\mathrm{~m}, 1 \mathrm{H}, \mathrm{H}_{11}\right), 5.58\left(\mathrm{~m}, 1 \mathrm{H}, \mathrm{H}_{10}\right)$, $5.82\left(\mathrm{dddd}, J=17.0,10.3,8.0,5.3 \mathrm{~Hz}, 1 \mathrm{H}, \mathrm{H}_{8}\right) .{ }^{13} \mathrm{C}-\mathrm{NMR}\left(\mathrm{CDCl}_{3}, 100 \mathrm{MHz}\right): \delta-4.3\left(\mathrm{q}, \mathrm{C}_{12}\right)$, $-4.2\left(\mathrm{q}, \mathrm{C}_{12}\right), 18.1\left(\mathrm{~s}, \mathrm{C}_{13}\right), 23.4\left(\mathrm{t}, \mathrm{C}_{5}\right), 25.9\left(\mathrm{q}, 3 \mathrm{C}_{14}\right), 34.0\left(\mathrm{t}, \mathrm{C}_{4}\right), 51.6\left(\mathrm{t}, \mathrm{C}_{6}\right), 58.2\left(\mathrm{t}, \mathrm{C}_{7}\right), 71.8$ $\left(\mathrm{d}, \mathrm{C}_{2}\right), 73.7\left(\mathrm{~d}, \mathrm{C}_{3}\right), 117.6\left(\mathrm{t}, \mathrm{C}_{9}\right), 119.4\left(\mathrm{t}, \mathrm{C}_{11}\right), 135.1\left(\mathrm{~d}, \mathrm{C}_{8}\right), 138.9$ (d, $\left.\mathrm{C}_{10}\right)$. IR (neat): 2928, 2857, 2790, 1643, 1462, 1361, 1253, 1101, 997, 916, 834, $772 \mathrm{~cm}^{-1}$. MS (EI, 70eV) $\mathrm{m} / \mathrm{z}$ (relative intensity): $281\left(\mathrm{M}^{+}, 4\right), 241$ (20), 240 (100), 224 (15), 185 (9), 150 (10), 122 (10), 110 (56), 108 (23), $73(28) .[\alpha]_{\mathrm{D}}{ }^{20}=+64.2\left(c \quad 0.67, \mathrm{CHCl}_{3}\right)$. HRMS: Found: $m / z$ 282.2257. Calcd for $\mathrm{C}_{16} \mathrm{H}_{32} \mathrm{NOSi}:(\mathrm{MH})^{+}, 282.2253$.

(8R,8aS)-8-(tert-Butyldimethylsilanyloxy)-3,5,6,7,8,8a-hexahydroindolizine $\quad(8))^{23 b}$ To a solution of piperidine $7\left(79 \mathrm{mg}, 0.28 \mathrm{mmol}, 1.0\right.$ equiv) in $\mathrm{CH}_{2} \mathrm{Cl}_{2}(10 \mathrm{~mL})$ at $0{ }^{\circ} \mathrm{C}$, (+)-camphorsulfonic acid (72 $\mathrm{mg}, 0.31 \mathrm{mmol}, 1.1$ equiv) was added. After $10 \mathrm{~min}$ at $0{ }^{\circ} \mathrm{C}$, the cooling bath was removed and Grubbs catalyst first generation was added by portions $(3 \times 8 \mathrm{mg}$, $0.035 \mathrm{mmol}, 12.5 \mathrm{~mol} . \%$ ), each addition was followed by $2 \mathrm{~h}$ reflux. The reaction mixture was then treated with $\mathrm{K}_{2} \mathrm{CO}_{3}$ and filtered. The solvent was removed in vacuo. The crude oil was purified by flash column chromatography on silica gel (chloroform/MeOH: 90/10) to give 8 (58 mg, $0.23 \mathrm{mmol}, 82 \%$ yield) as a yellow oil. ${ }^{1} \mathrm{H}-\mathrm{NMR}\left(\mathrm{C}_{6} \mathrm{D}_{6}, 400 \mathrm{MHz}\right): \delta 0.05\left(\mathrm{~s}, 3 \mathrm{H}, \mathrm{H}_{9}\right)$, $0.07\left(\mathrm{~s}, 3 \mathrm{H}, \mathrm{H}_{9}\right), 0.99$ (s, 9H, H$\left.{ }_{11}\right), 1.27(\mathrm{~m}, 1 \mathrm{H}), 1.41(\mathrm{~m}, 1 \mathrm{H}), 1.59$ (m, 1H), 1.89 (ddd, J= 12.2, 7.9, $4.0 \mathrm{~Hz}, 1 \mathrm{H}), 2.25$ (ddd, $\left.J=11.6,11.6,2.9 \mathrm{~Hz}, 1 \mathrm{H}, \mathrm{H}_{5}\right), 2.72\left(\mathrm{dd}, J=11.2,4.8 \mathrm{~Hz}, 1 \mathrm{H}, \mathrm{H}_{8 \mathrm{a}}\right.$ ), $3.04\left(\mathrm{~m}, 1 \mathrm{H}, \mathrm{H}_{5}\right.$ ), 3.13 (dddd, $\left.J=12.5,6.5,2.0,2.0 \mathrm{~Hz}, 1 \mathrm{H}, \mathrm{H}_{3}\right), 3.54$ (dddd, $J=12.5,3.9,1.9,1.9$ $\mathrm{Hz}, 1 \mathrm{H}, \mathrm{H}_{3}$ ), 3.60 (ddd, $\left.J=10.2,8.8,4.4 \mathrm{~Hz}, 1 \mathrm{H}, \mathrm{H}_{8}\right), 5.69$ (ddd, $J=6.2,4.0,2.1 \mathrm{~Hz}, 1 \mathrm{H}, \mathrm{H}_{1}$ ), 
$6.18\left(\mathrm{~m}, 1 \mathrm{H}, \mathrm{H}_{2}\right) .{ }^{13} \mathrm{C}-\mathrm{NMR}\left(\mathrm{C}_{6} \mathrm{D}_{6}, 100 \mathrm{MHz}\right): \delta-4.5\left(\mathrm{q}, \mathrm{C}_{9}\right),-4.1\left(\mathrm{q}, \mathrm{C}_{9}\right), 18.3\left(\mathrm{~s}, \mathrm{C}_{10}\right), 25.2(\mathrm{t}$, $\left.\mathrm{C}_{6}\right), 26.1\left(\mathrm{q}, 3 \mathrm{C}_{11}\right), 35.1\left(\mathrm{t}, \mathrm{C}_{7}\right), 49.1\left(\mathrm{t}, \mathrm{C}_{5}\right), 58.3\left(\mathrm{t}, \mathrm{C}_{3}\right), 72.5\left(\mathrm{~d}, \mathrm{C}_{8}\right), 74.8\left(\mathrm{~d}, \mathrm{C}_{8 \mathrm{a}}\right), 128.9\left(\mathrm{~d}, \mathrm{C}_{1}\right)$, 131.5 (d, $\mathrm{C}_{2}$ ). IR (neat): 2931, 2886, 2857, 2778, 1467, 1368, 1253, 1148, 1095, 839, $775 \mathrm{~cm}^{-1}$. MS (CI, $\left.\mathrm{CH}_{4}\right) \mathrm{m} / z$ (relative intensity): $254\left(\mathrm{MH}^{+}, 100\right), 144$ (3), 120 (24), 111 (3). $[\alpha]_{\mathrm{D}}{ }^{20}=-88.9\left(c 0.70, \mathrm{C}_{6} \mathrm{H}_{6}\right)$.

\section{Acknowledgements}

Johnson \& Johnson is greatly acknowledged for financial support (Focus Giving Program to J. Cossy)

\section{References}

1. Guengerich, F. P.; DiMari, S. J.; Broquist, H. P. J. Am. Chem. Soc. 1973, 95, 2055.

2. (a) Colegate, S. M.; Dorling, P. R.; Huxtable, C. R. Aust. J. Chem. 1979, 32, 2257. (b) Ermayanti, T. M.; McComb, J. A.; O’Brien, P. A. Phytochemistry 1994, 36, 313.

3. Tamerler, C.; Kesharaz, T. Biotechnol. Lett. 1999, 21, 501.

4. Liao, Y. F.; Lal, A.; Moreman, K. W. J. Biol. Chem. 1996, 271, 28348.

5. (a) Elbein, A. D.; Solf, R.; Dorling, P. R.; Vosbeck, K. Proc. Natl. Acad. Sci. U.S.A. 1981, 78, 7393. (b) Kaushal, G. P.; Szumilo, T.; Pastuszak, I.; Elbein, A. D. Biochemistry 1990, 29, 2168. (c) Pastuszak, I.; Kaushal, G. P.; Wall, K. A.; Pan, Y. T.; Sturm, A.; Elbein, A. D. Glycobiology 1990, 1, 71 .

6. (a) Goss, P. E.; Baker, M. A.; Carver, J. P.; Dennis, J. W. Clin. Cancer Res. 1995, 1, 935.

(b) Goss, P. E.; Reid, C. L.; Bailey, D.; Dennis, J. W. Clin. Cancer Res. 1997, 3, 1077.

7. Dennis, J. W. Cancer Res. 1986, 46, 5131.

8. White, S. L.; Schweitzer, K.; Humphries, M. J.; Olden, K. Biochem. Biophys. Res. Commun. 1988, 150, 615 .

9. Mezher, H. A.; Hough, L.; Richardson, A. C. J. Chem. Soc., Chem. Commun. 1984, 447.

10. Fleet, G. W. J.; Gouh, M. J.; Smith, P. W. Tetrahedron Lett. 1984, 25, 1853.

11. Suami, T.; Tadano, K.; Iimura, Y. Chem. Lett. 1984, 513.

12. Adams, C. E.; Walker, F. J.; Sharpless, K. B. J. Org. Chem. 1985, 50, 420.

13. For review see: (a) Nemr, A. E. Tetrahedron 2000, 56, 8579. (b) Pyne, S. G. Current Organic Synthesis 2005, 2, 39.

14. Guo, H.; O’Doherty, G. A. Org. Lett. 2006, 8, 1609.

15. Bennett, R. B.; Choi, J. R.; Montgomery, W. D.; Cha, J. K. J. Am. Chem. Soc. 1989, 111, 2580.

16. Pearson, W. H.; Ren, Y.; Powers, J. D. Heterocycles 2002, 58, 421. 
17. For comprehensive reviews, see: (a) Cossy, J.; Gomez Pardo, D. Chemtracts 2002, 15, 579. (b) Cossy, J.; Gomez Pardo, D. Targets in Heterocyclic Systems 2002, 6, 1.

18. For reviews, see: (a) Fürstner, A. Angew. Chem., Int. Ed. Engl. 2000, 39, 3012. (b) Grubbs R. G.; Chang, S. Tetrahedron 1998, 54, 4413. (c) Armstrong, S. J. Chem. Soc., Perkin Trans. 1 1998, 371. (d) Schuster, M. ; Blechert, S. Angew. Chem., Int. Ed. Engl. 1997, 36, 2036.

19. Bejjani, J.; Chemla, F.; Audouin, M. J. Org. Chem. 2003, 68, 9747.

20. (a) Andrès, J. M.; Pedrosa, R.; Pérez, A.; Pérez-Encabo, A. Tetrahedron 2001, 57, 8521. (b) Ma, D.; Pan, Q.; Han, F. Tetrahedron Lett. 2002, 43, 9401.

21. (a) Cossy, J.; Dumas, C.; Michel, P.; Gomez Pardo, D. Tetrahedron Lett. 1995, 36, 549. (b) Cossy, J.; Dumas, C.; Gomez Pardo, D. Synlett 1997, 905. (c) Cossy, J.; Dumas, C.; Gomez Pardo, D. Bioorg. Med. Chem. Lett. 1997, 7, 1343. (d) Wilken, J.; Kossenjans, M.; Saak, W.; Haase, D.; Pohl, S.; Martens, J. Liebigs Ann. 1997, 573. (e) Langlois, N.; Calvez, O. Synth. Commun. 1998, 28, 4471. (f) Davis, P. W.; Osgood, S. A.; Hébert, N.; Sprankle, K. G.; Swayze, E. E. Biotechnol. Bioeng. 1999, 61, 143. (g) Cossy, J.; Dumas, C.; Gomez Pardo, D. Eur. J. Org. Chem. 1999, 1693. (h) Michel, P.; Rassat, A. J. Org. Chem. 2000, 65, 2572. (i) Cossy, J.; Mirguet, O.; Gomez Pardo, D. Synlett 2001, 1575. (j) Brandi, A.; Cicchi, S.; Paschetta, V.; Gomez Pardo, D.; Cossy, J. Tetrahedron Lett. 2002, 43, 9357. (k) Deyine, A.; Delcroix, J.-M.; Langlois, N. Heterocycles 2004, 64, 207. (1) Déchamps, I.; Gomez Pardo, D.; Karoyan, P.; Cossy, J. Synlett 2005, 1170. (m) Roudeau, R.; Gomez Pardo, D.; Cossy, J. Tetrahedron 2006, 62, 2388. (n) Mena, M.; Bonjoch, J.; Gomez Pardo, D.; Cossy, J. J. Org. Chem. 2006, 71, 5930.

22. Schwab, P.; France, M. B.; Ziller, J. W.; Grubbs, R. H. Angew. Chem., Int. Ed. Engl. 1995, 34, 2039.

23. (a) Mukai, C.; Sugimoto, Y.-I.; Miyazawa, K.; Yamaguchi, S.; Hanaoka, M.; J. Org. Chem. 1998, 63, 6281. (b) Buschmann, N.; Rückert, A.; Blechert, S. J. Org. Chem. 2002, 67, 4325. 